\title{
Controlled Indentation Flaws for the Construction of Toughness and Fatigue Master Maps
}

\author{
R. F. Cook \\ University of New South Wales, Kensington, N. S. W. 2033, Australia \\ and \\ B. R. Lawn \\ National Bureau of Standards, Gaithersburg, MD 20899
}

\begin{abstract}
Accepted: August 30, 1984
A simple and economical procedure for accurate determinations of toughness and lifetime parameters of ceramics is described. Indentation flaws are introduced into strength test pieces, which are then taken to failure under specified stressing and environmental conditions. By controlling the size of the critical flaw, via the contact load, material characteristics can be represented universally on "master maps" without the need for statistical considerations.

This paper surveys both the theoretical background and the experimental methodology associated with the scheme. The theory is developed for "point" flaws for dynamic and static fatigue, incorporating load explicitly into the analysis. A vital element of the fracture mechanics is the role played by residual contact stresses in driving the cracks to failure. Experimental data on a range of Vickers-indented glasses and ceramics are included to illustrate the power of the method as a means of graphic materials evaluation. It is demonstrated that basic fracture mechanics parameters can be measured directly from the slopes, intercepts and plateaus on the master maps, and that these parameters are consistent, within experimental error, with macroscopic crack growth laws.
\end{abstract}

Key words: ceramics; fatigue; indentation flaw; lifetime prediction; master mäps; materials evaluation; strength; toughness; universal curves.

\section{Introduction}

The increasing use of glasses and ceramics as structural materials has prompted the development of new and accurate techniques for evaluating intrinsic fracture

\footnotetext{
About the Authors, Paper: A graduate student in the Department of Applied Physics in Australia's University of New South Wales, R. F. Cook is currently working under B. R. Lawn, a physicist in the NBS Center for Materials Science. Their paper will also appear in a forthcoming ASTM Special Technical Publication, Methods for Assessing the Structural Reliability of Brittle Materials, S. W. Freiman, editor. Funding for the work was provided by the Australian Research Grants Committee and the U.S. Office of Naval Research (Metallurgy and Ceramics Program).
}

parameters. Chief among these parameters are the fracture toughness, $K_{\mathrm{c}}$, and the crack velocity exponent, $n$, which respectively characterize the equilibrium and $\mathrm{ki}$ netic crack growth responses. In the context of brittle design it is essential to achieve an adequate level of precision in such parameter evaluations. This is particularly so in the consideration of component integrity under sustained stresses and chemical environments, where apparently minor uncertainties can translate into order-of-magnitude discrepancies in lifetime predictions.

A standard method of determining basic fracture parameters for design is to measure the strengths of representative test specimens in flexure. However, for specimens with typically as-received or as-prepared surfaces, these strengths depend not only on the intrinsic material properties but on the flaw distributions as well. It is then not possible to investigate these two elements of the 
problem in any truly independent way. Evaluation of material parameters becomes a mere exercise in statistical data manipulation, with little or no physical insight into the nature of the critical flaws responsible for failure $[1-2]{ }^{1}$ This probabilistic approach makes it difficult to assess the relative merits of different materials from the standpoint of intrinsic properties alone.

A controlled-flaw technique which effectively eliminates the statistical component from strength testing has been described in a series of recent articles [3-12]. A single dominant flaw of predetermined size and geometry is introduced into the prospective tensile surface of each specimen using a standard diamond indenter. The specimens are then stressed to failure in the usual way. With the indentation and flexure testing conditions held fixed, any variations in the strength behavior can be taken as direct reflections of the intrinsic material response. The only need for statistical treatments then resides in the trivial accountability of random scatter in the data. Quite apart from the ensuing improvements in data reproducibility, the indentation procedure confers several advantages in strength analysis: (i) greater specimen economy, (ii) because the location of the critical flaw is predetermined, closer observation of the fracture mechanics to failure, and (iii) a reasonable simulation of the damage processes that are responsible for a great many brittle failures [13-15]. One apparent complication attending the technique is the existence of a strong residual contact field about the elastic/plastic deformation zone, necessitating the incorporation of additional terms in the governing stress intensity factor. However, closed-form solutions of the fracture mechanics formulations are now available for both equilibrium [4] and kinetic [16] conditions of failure; analytical determinations of toughness and fatigue parameters from the strength data may accordingly be made in as straightforward a manner as for "Griffith" flaws without the residual stress term.

The capacity to control the scale of the critical flaw via the indentation load is a potent tool in the investigation of material fracture properties. The load actually replaces initial crack size as a variable in the fracture equations, thereby eliminating the need for onerous measurements of crack dimensions (although some observations of crack growth are useful for confirming the validity of the theory) [15]. Size effects in the micromechanics may then be studied systematically: important changes in the nature of low-load contact flaws have been thus revealed on reducing the crack size to

\footnotetext{
'Figures in brackets indicate literature references at the end of this paper.
}

the scale of the deformation zone [17] or of the microstructure [18]. Systematic variations in the load dependence of indentation-strength characteristics can also be used to evaluate pre-existing stress states in britthe materials, e.g., in tempered glass [19]. Again, some materials may produce ill-defined indentation patterns outside certain ranges of flaw size, or be restricted in specimen dimensions, in which case the geometrical requirements of standard strength-testing procedures may make it impossible to operate at a single contact load. The theoretical analysis allows one to compensate for any such changes in the working contact conditions, effectively reducing all data to an "equivalent" load.

This paper illustrates a procedure for representing the intrinsic strength properties of brittle materials on an indentation "master map." A suitable "normalization" scheme incorporating indentation load into the plotting coordinates allows for the reduction of all inert and fatigue strength data on to "universal" curves for the various test materials. In this sense the scheme is reminiscent of that developed earlier by Mould and Southwick [20], except that their use of relatively ill-defined abrasion flaws necessitated a totally empirical approach in the data reduction. On our master map the position of a given curve may be taken as a graphic indicator of the intrinsic toughness and fatigue susceptibility. Quantitative determinations may accordingly be made of $K_{c}$ and $n$ without recourse to statistically based theories of strength.

\section{Background Theory}

\section{Stress Intensity Factor for Indentation Cracks}

The starting point in the analysis is the stress intensity factor for an indentation crack of characteristic dimension $c$ produced at peak contact load $P$ and subjected to subsequent applied tensile stress $\sigma_{\mathrm{a}}$. For "point" flaws produced by axially loaded indenters the general form of this stress intensity factor is [4]

$$
K=\chi P / c^{3 / 2}+\psi \sigma_{\mathrm{a}} c^{1 / 2}
$$

where $\chi$ and $\psi$ are dimensionless parameters. The second term in eq (1) is the familiar contribution from the applied field; $\psi$ depends only on crack geometry, here assumed to be essentially "penny-like" [21]. The first term is the contribution from the residual contact field; for materials which deform irreversibly by a constant volume process

$$
\chi=\xi(E / H)^{1 / 2}
$$


approximately [22], where $E$ is Young's modulus, $H$ is hardness and $\xi$ is a numerical constant.

In the event of any pre-existent stress acting on the crack a third term would have to be included in eq (1) $[4,9]$. Other than to note that this potential complication needs to be heeded when preparing the surfaces of test specimens we shall consider it no further in our mathematical derivations.

\section{Equilibrium Solutions: Inert Strengths}

Equilibrium conditions of crack growth are closely realized experimentally by testing in an inert environment. In terms of fracture mechanics notation the criterion for equilibrium is that $K=K_{c}$; if $d K / d c<0$, the equilibrium is stable, if $d K / d c>0$, it is unstable. Now it is evident from eq (1) that $K$ for given values of $P$ and $\sigma_{\mathrm{a}}$ passes through a minimum in its functional dependence on $c$; thus at subcritical configurations $K(\mathrm{~min})<K_{\mathrm{c}}$ there is a stable and an unstable equilibrium, to the left and to the right of the minimum, respectively [16]. In an inert strength test, $\sigma_{a}$ is increased steadily until these two equilibria merge at $d K / d c=0$, which defines the critical variables

$$
\begin{gathered}
\sigma_{\mathrm{m}}=3 K_{\mathrm{c}} / 4 \psi c_{\mathrm{m}}{ }^{1 / 2} \\
c_{\mathrm{m}}=\left(4 \chi P / K_{\mathrm{c}}\right)^{2 / 3}
\end{gathered}
$$

at which crack growth proceeds without limit. We may note that any relaxation of the residual stress field, as reflected in a reduction in $\chi$ (or, more specifically, in $\xi$ in eq (2)), will cause $\sigma_{\mathrm{m}}$ to expand and $c_{\mathrm{m}}$ thence to contract.

It can be shown that the ideal indentation crack is in a state of equilibrium immediately after completion of the contact cycle [22]. The size of this crack is found by setting $\sigma_{\mathrm{a}}=0, K=K_{\mathrm{c}}$ in eq (1);

$$
c_{0}=\left(\chi P / K_{\mathrm{c}}\right)^{2 / 3} \text {. }
$$

From eq (3b) we have $c_{0} \approx 0.40 c_{\mathrm{m}}$. On subsequently applying the tensile stress, the crack extends stably from $c_{0}$ to $c_{\mathrm{m}}$, whence spontaneous failure ensues at $\sigma_{\mathrm{a}}=\sigma_{\mathrm{m}}$ [4]. In reality, deviations from this ideal behavior are observed; relaxation effects can cause $c_{\mathrm{m}}$ to contract, as already mentioned, and subcritical, moisture-assisted crack extension within the residual contact field can cause $c_{0}$ to expand, to $c_{0}^{\prime}$ say. Nevertheless, unless the condition $c_{0}^{\prime} \leqslant c_{\mathrm{m}}$ is violated, some precursor crack growth will still precede failure, in which case $\sigma_{\mathrm{m}}$ remains a measure of the inert strength.

Equation (3) may then be conveniently rearranged to eliminate all terms in crack size, and then combined with eq (2) to yield

$$
\sigma_{\mathrm{m}} P^{1 / 3}=(3 / 4 \psi)(1 / 4 \xi)^{1 / 3}\left[(H / E)^{1 / 8} K_{\mathrm{c}}\right]^{4 / 3} .
$$

This expression conveniently relates the test variables on the left side to the material properties, primarily the toughness, on the right side. We emphasize once more that this formulation is contingent on the absence of all spurious pre-present stresses.

\section{Kinetic Solutions: Dynamic Fatigue}

When cracks are exposed to moisture or other interactive environmental species, extension can occur in the subcritical region, $K<K_{\mathrm{c}}$. The major characteristic of this kind of extension is its rate dependence, which is in turn highly sensitive to the crack driving force. The basic equation of kinetic fracture accordingly takes the form of a crack velocity $v(K)$. In the interest of obtaining closed-form solutions to the ensuing fracture mechanics relations we choose the empirical power-law function [23]

$$
v=v_{0}\left(K / K_{\mathrm{c}}\right)^{n}
$$

where $v_{0}$ and $n$ are material/environment parameters. Materials with lower values of $n$ are said to be more "susceptible" to kinetic crack growth effects.

The most practical loading arrangement for the systematic study of rate effects in strength properties is that of "dynamic fatigue," in which the time differential of stress is held fixed up to the point of failure, i.e., $\dot{\sigma}_{\mathrm{a}}=\sigma_{\mathrm{a}} / t=$ const. We may thus combine eqs (1) and (6) to obtain a differential equation for this stressing configuration,

$$
\mathrm{d} c / \mathrm{d} t=v_{0}\left[\chi P / K_{\mathrm{c}} \mathrm{c}^{3 / 2}+\psi \dot{\sigma}_{\mathrm{a}} c^{1 / 2} t / K_{\mathrm{c}}\right]^{n} .
$$

This equation has to be solved at given $P$ and $\dot{\sigma}_{\mathrm{a}}$ for the time to take the crack from its initial configuration, $K=K\left(c_{0}^{\prime}\right)$, to its final configuration, $K=K_{\mathrm{c}}$, at which point the stress level defines the dynamic fatigue strength, $\sigma_{a}=\sigma_{\mathrm{f}}[16]$

$$
\sigma_{\mathrm{f}}=\left(\lambda^{\prime} \dot{\sigma}_{\mathrm{a}}\right)^{1 /\left(n^{\prime}+1\right)}
$$

where

$$
\begin{gathered}
n^{\prime}=3 n / 4+1 / 2 \\
n^{\prime}=\left(2 \pi n^{\prime}\right)^{1 / 2} \sigma_{\mathrm{m}}{ }^{{ }^{\prime}} c_{\mathrm{m}} / v_{0} .
\end{gathered}
$$


The solution in eq (8) is identical in form to that for "Griffith" flaws $(\chi=0)$ [23]. However, the slopes and intercepts from a linear plot of $\log \sigma_{f}$ against $\log \dot{\sigma}_{\mathrm{a}}$ are very different in the two instances. In the present case $(\chi \neq 0), n^{\prime}$ and $\lambda^{\prime}$ may be regarded as "apparent" fatigue parameters, in the sense that transformation equations are required to convert these to "true" crack velocity exponent and coefficient terms. Thus, eq (9a) may be inverted to obtain $n$ directly from $n^{\prime}$, and eq (9b) similarly (in conjunction with measured values of $\sigma_{\mathrm{m}}$ and $c_{\mathrm{m}}$ ) to obtain $v_{0}$ from $\lambda^{\prime}$. It is again seen that initial crack size does not enter the results, as long as the condition $c_{0}^{\prime} \leqslant c_{\mathrm{m}}$ remains operative [9].

Implicit in the derivation of eq (8) is the usual assumption that the prospective test surfaces are free of spurious stresses. The introduction of such stresses leads to nonlinearities in the dynamic fatigue plotting scheme, thereby destroying the basis for the above analysis $[9,10]$.

It is convenient at this point to incorporate the indentation load as a working test variable into the dynamic fatigue relations. Whereas $n^{\prime}$ in eq (9a) is independent of all test variables, $\lambda^{\prime}$ in eq (9b) can be expressed as an explicit function of $P$ via the quantities $\sigma_{\mathrm{m}}$ and $c_{\mathrm{m}}$ in eq (3). In this way we may write

$$
\lambda^{\prime}=\lambda_{p}^{\prime} / P^{\left(n^{\prime}-2\right) / 3}
$$

where $\lambda_{P}^{\prime}$ is a modified intercept term, totally independent of $P$, given by

$$
\lambda_{P}^{\prime}=\left(2 \pi n^{\prime}\right)^{1 / 2}\left(3 K_{\mathrm{c}} / 4 \psi\right)^{n^{\prime}}\left(K_{\mathrm{c}} / 4 \chi\right)^{\left(n^{\prime}-2\right) / 3} / v_{0}
$$

Equation (10) tells us that fatigue data obtained on one material but using different indentation loads will fall on different straight lines, mutually translated but without change of slope. Now by inserting eq (10) into eq (8) we may appropriately modify the dynamic fatigue relation, thus

$$
\sigma_{\mathrm{f}} P^{1 / 3}=\left(\lambda_{P}^{\prime} \dot{\sigma}_{\mathrm{a}} P\right)^{1 /\left(n^{\prime}+1\right)}
$$

such that by plotting $\log \left(\sigma_{\mathrm{f}} P^{1 / 3}\right)$ against $\log \left(\dot{\sigma}_{\mathrm{a}} P\right)$ all data should fall on to a universal fatigue curve. This plot would, of course, cut off at a limiting level on the ordinate corresponding to the inert strength plateau defined in eq (5). The procedure for evaluating crack velocity parameters from the slopes and intercepts of such representations is the same as before, but with eq (10) serving as an intermediary to eq (9).

\section{Kinetic Solutions: Static Fatigue}

Of more practical interest from a design standpoint is the issue of component lifetime under fixed stress rather than stress rate. Ideally, it would seem desirable to formulate a universal static fatigue relation in direct analogy to eq (12) retaining, as far as possible, the same adjustable parameters. Lifetime predictions could then be made from dynamic fatigue data alone, without having to resort to delayed failure experiments. This formulation may be achieved in two steps. First, eliminate stressing rate in favor of time to failure, $\dot{\sigma}_{\mathrm{a}}=\sigma_{\mathrm{f}} / t_{\mathrm{f}}$. This step introduces the lifetime concept without yet altering the status of eq (12) as a dynamic fatigue relation. Then, convert to equivalent static fatigue variables by replacing $\sigma_{f}$ with $\sigma_{A}$, i.e., the level of the invariant applied stress, and $t_{\mathrm{f}}$ with $\left(n^{\prime}+1\right) t_{\mathrm{f}}[16]$. The resulting static fatigue relation is

$$
t_{\mathrm{f}} / P^{2 / 3}=\lambda_{P}^{\prime} /\left(n^{\prime}+1\right)\left(\sigma_{\mathrm{A}} P^{1 / 3}\right)^{n^{\prime}} .
$$

We reiterate here, at the risk of laboring the point, that the variables $P, \sigma_{\mathrm{A}}$ and $t_{\mathrm{f}}$ in eq (13) relate to prospective static fatigue conditions, whereas the parameters $n^{\prime}$ and $\lambda_{\mathrm{p}}^{\prime}$ are adjustables, as defined by eqs ( 9 ) and (10), to be determined from dynamic fatigue data.

\section{Experimental}

\section{Materials Selection and Preparation}

The materials in this study were chosen in accordance with two major criteria: first, they should cover a range of toughness and crack velocity characteristics, as determined by independent fracture techniques; second, they should be of some technical importance. Table 1 lists these materials and their pertinent properties.

All specimens were prepared in the usual manner for strength testing. However, particular attention was paid to surface preparation, bearing in mind our repeated assertion that pre-existing stress states can greatly influence the interpretation of strength data. The glass specimens were therefore annealed [19] and the ceramics surface-polished to a mirror finish with diamond paste [10] to ensure removal of any such stresses.

\section{Indentation and Strength Testing Procedure}

All specimens were routinely indented centrally along their length using a Vickers diamond pyramid indenter to produce dominant flaws for the subsequent 
Table 1. Materials used in this study.

\begin{tabular}{|c|c|c|c|c|c|c|c|}
\hline \multirow[b]{2}{*}{$\begin{array}{c}\text { Materials } \\
\text { (sources footnoted) }\end{array}$} & \multicolumn{4}{|c|}{ Independent parameters } & \multicolumn{3}{|c|}{ Indentation parameters } \\
\hline & $\begin{array}{c}E \\
\mathrm{GPa}\end{array}$ & $\begin{array}{c}H \\
\mathrm{GPa}\end{array}$ & $\begin{array}{c}K_{\mathrm{c}} \\
\mathrm{MPa} \mathrm{m}\end{array}$ & $n$ & $\begin{array}{c}K_{\mathrm{c}} \\
\mathrm{MPa} \mathrm{m}^{\mathrm{t}}\end{array}$ & $n$ & $\begin{array}{l}\log v_{0} \\
\mathrm{~ms}^{-1}\end{array}$ \\
\hline Soda-Lime Glass ${ }^{a}$ & 70 & 6.6 & $0.74^{*}$ & $16-19^{*}$ & 0.97 & 18 & -1.6 \\
\hline Borosilicate Glass ${ }^{b}$ & 89 & 6.5 & $0.77^{*}$ & $31-37^{*}$ & 1.2 & 36 & 1.6 \\
\hline Fused Silica ${ }^{c}$ & 72 & 7.6 & $0.81^{*}$ & $38^{*}$ & 1.2 & 44 & 2.2 \\
\hline Synroc ${ }^{d}$ & 190 & 10.3 & 1.9 & - & 1.8 & 35 & 0.2 \\
\hline P.Z.T. ${ }^{\mathrm{e}}$ & 88 & 3.1 & 0.87 & - & 1.0 & 43 & -0.5 \\
\hline Alumina $^{f}$ & 400 & 16 & 4.4 & $46^{*}$ & 3.8 & 59 & 1.7 \\
\hline Silicon Carbide ${ }^{8}$ & 435 & 24 & $4.1^{*}$ & $118^{*}$ & 3.7 & 222 & 8.4 \\
\hline Glass Ceramic ${ }^{\text {h }}$ & 108 & 8.4 & $2.5^{*}$ & $63, * 84^{*}$ & 2.2 & 117 & 5.0 \\
\hline \multicolumn{4}{|c|}{$\begin{array}{l}\text { DDeterminations by other workers. (See references, below). } \\
\text { aSchott-Ruhrglas GMBH }[11,31,32] \text {. } \\
\text { 'Schott-Ruhrglas GMBH }[11,31,32] \text {. } \\
\text { 'Schott-Ruhrglas GMBH }[31,33] \text {. }\end{array}$} & \multicolumn{4}{|c|}{ 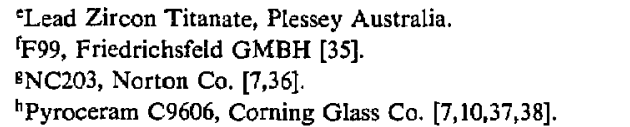 } \\
\hline
\end{tabular}

failure tests. The Vickers geometry was chosen both for its proven capacity to produce well-defined radial crack patterns and for its general availability in hardness testing facilities. The glasses were indented at several loads, ranging from 0.05 to $100 \mathrm{~N}$, whereas the ceramics were each indented at single loads, 10,20 , or $100 \mathrm{~N}$. In all cases the radial cracks extended well beyond the central hardness impression, but never to a length in excess of one tenth the specimen thickness.

The indented specimens were then broken in fourpoint flexure [24] in a universal testing machine at constant crosshead speed. Care was taken to center the indentation on the tension side, with one set of radial cracks aligned normal to the long axis. The breaking loads were recorded using conventional strain gage and piezoelectric load cells [10], and the corresponding rupture stresses thence evaluated from simple beam theory. Inert strengths, $\sigma_{\mathrm{m}}$, were measured in dry nitrogen or argon or silicone oil environments, with the crosshead running at its maximum speed. Dynamic fatigue strengths, $\sigma_{\mathrm{f}}$, were measured in distilled water over the allowable range of crosshead speeds. At least six specimens were broken in each strength evaluation, from which means and standard deviations were computed.

\section{Measurement of Critical Crack Dimensions}

For the purpose of confirming the necessary condition that the initial crack size $c_{0}^{\prime}$ should never exceed the instability value $c_{\mathrm{m}}$ for equilibrium failure, and for verifying certain aspects of the fatigue solutions presented earlier, an optical examination of representative critical indentations is recommended. The technique used here was to place three indentations instead of one on a given test surface, and then take the specimen to failure under inert conditions [10]. On the understanding that all three indentations must have had nearly identical growth histories, the procedure leaves two "dummies" in the broken test piece from which to measure the required crack dimensions. The Vickers geometry proves particularly useful in this technique, for while the set of radial cracks perpendicular to the tensile direction provides a measure of $c_{m}$, the set parallel to this same direction remains free of external stress and hence provides a measure of $c_{0}^{\prime}$.

In all materials studied in this work some precursor crack growth was indeed found to occur prior to failure.

\section{Results}

\section{Inert Strengths and Toughness}

In this section we begin by examining the dependence of inert strength on indentation load for the three glasses studied. With this dependence established, we then investigate how the inert strength data may be reduced to a composite toughness parameter for all of the test materials.

Figure 1 accordingly shows $\sigma_{\mathrm{m}}$ as a function of $P$ for the glasses. The straight lines are best fits of slope $-1 / 3$ in logarithmic coordinates, as per eq (5). This same dependence has been confirmed elsewhere for several other brittle materials $[7,18,25,26]$.

Values of the composite parameter $\sigma_{\mathrm{m}} P^{1 / 3}$ are thus 


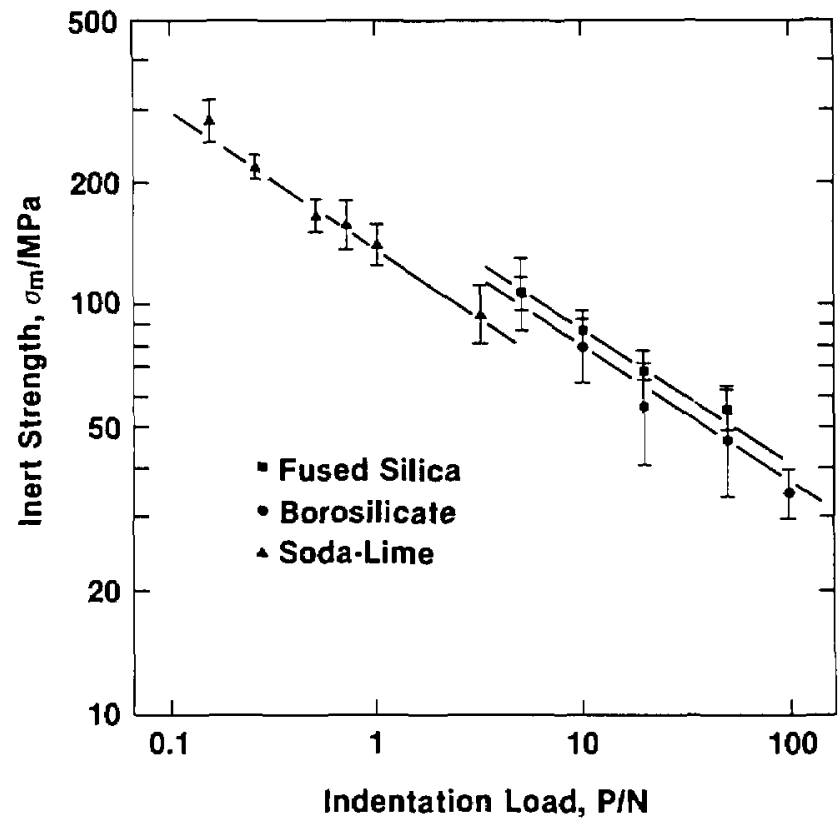

Figure 1-Inert strength as function of indentation load for the silicate glasses. (Data courtesy of T. P. Dabbs).

evaluated for each of the glasses and ceramics, and are plotted as a function of $(H / E)^{1 / B} K_{\mathrm{c}}$ (from table 1) in figure 2 . The straight line is a fit of logarithmic slope $4 / 3$ in accordance with eq (5), using a "calibration" value $(3 / 4 \psi)(1 / 4 \xi)^{1 / 3}=2.02$ from an earlier, more comprehensive study [7]. The trends in figure 2 appear to be in reasonable accord with prediction, although some deviations are evident, particularly for the fused silica and borosilicate glasses. Estimates of the "indentation

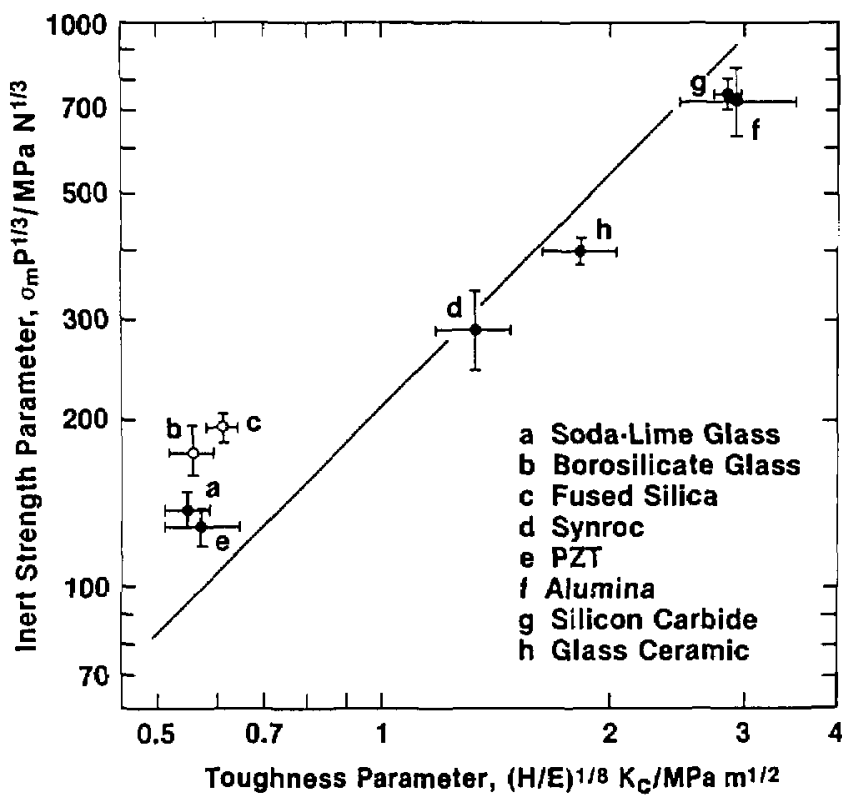

Figure 2-Inert strength parameter $\sigma_{\mathrm{m}} P^{1 / 3}$ as function of toughness parameter $(H / E)^{1 / 8} K_{\mathrm{c}}$ for the glasses and ceramics. toughness" obtained directly from $\sigma_{\mathrm{m}} P^{1 / 3}$ by inverting eq (5) are included in table 1 for comparison with the independently determined values.

\section{Dynamic Fatigue and Crack Velocity Parameters}

We consider now the dynamic fatigue responses, again beginning with the glasses to examine the functional influence of contact load, and outline the procedure for determining the exponent and coefficient in the crack velocity function.

Figure 3 shows these responses for the glass compositions in water. The straight lines drawn through individual sets of data at fixed $P$ are best fits to eq (8), regressed for each glass on all the data consistent with the intercept relation eq (10). Thus we obtain families of lines of constant slope, with systematic displacements to lower strength levels with increasing load. Analogous plots are shown in figure 4 for the five ceramics in the same water environment, but now for a single load ineach case. The inert strength limits are included in all plots as a reference baseline for assessing the degrees of fatigue.

From the regressed slopes and intercepts we obtain values of the apparent fatigue parameters $n^{\prime}$ and $\lambda^{\prime}$ in eq (8). Inversion of eq (9) (together with the inert strength data) then allows us to evaluate the true crack velocity parameters, $n$ and $v_{0}$. These evaluations are summarized in table 1; comparisons may be made in this tabulation with independent measurements of the crack velocity exponent.

\section{Master Maps}

We have set the base for determining universal fracture curves for the materials studied, and thence to construct master maps. We do this for dynamic and static fatigue conditions in turn.

The presentation of the dynamic fatigue results on a single master map requires conversion of all data to appropriate load-adjusted variables $\sigma_{\tilde{f}} P^{1 / 3}$ and $\dot{\sigma}_{\mathrm{a}} P$ in eq (12). Figure 5, an appropriate composite of all data thus converted from figures 3 and 4 (but with error bars omitted for clarity), is such a map. Each material is now conveniently represented by a universal curve, independent of the contact loads used to obtain the data. The curves plotted in this diagram represent numerical solutions of the basic fatigue differential equation, eq (7), obtained for the ranges of $P$ and $\dot{\sigma}_{\mathrm{a}}$ covered experimentally for each material, using the inert and kinetic parameters already determined along with the measured 

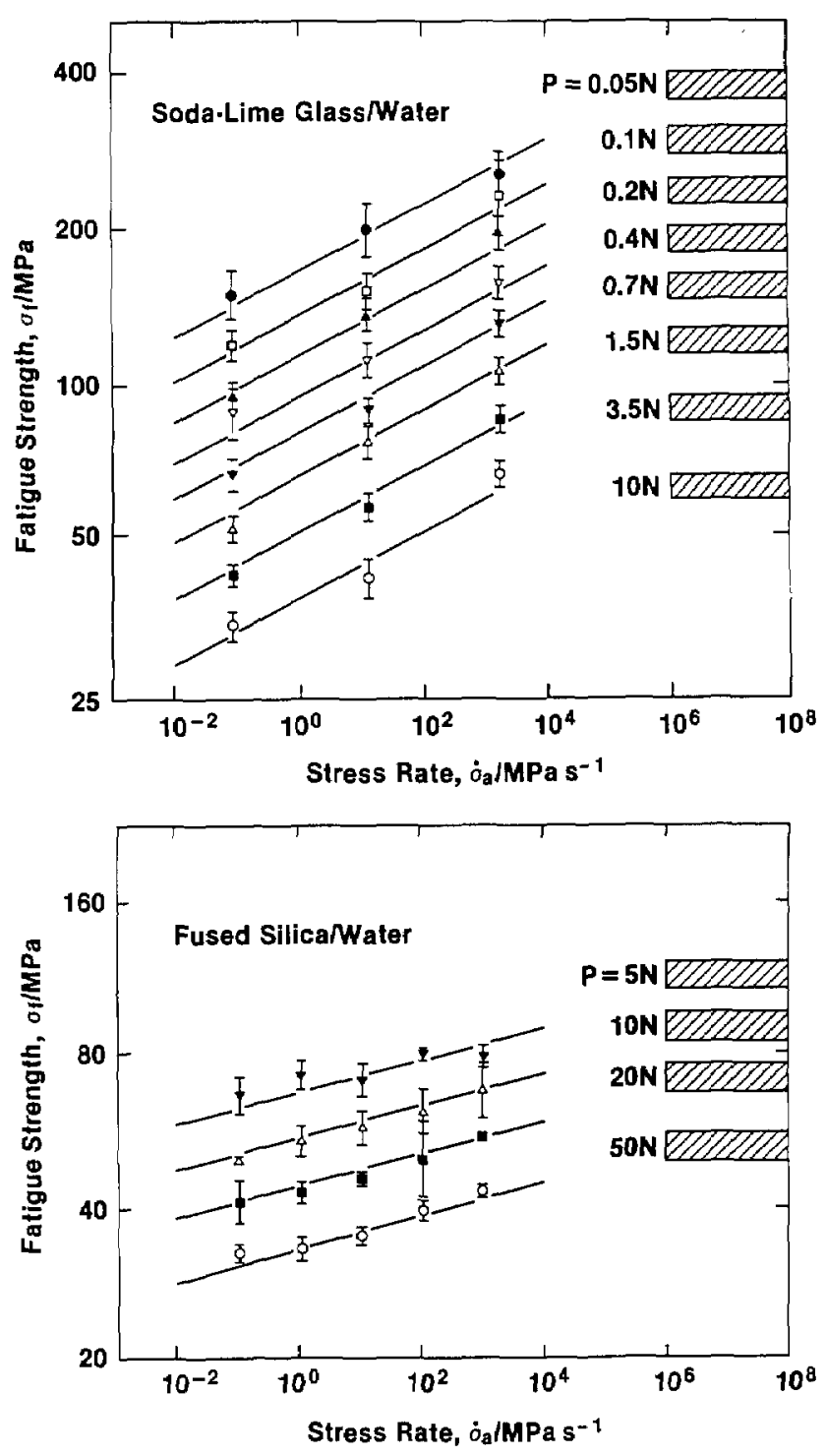

initial crack sizes [10]. The fact that the curves regenerated in this way are effectively coincident with the data is, of course, no real surprise, since the regression analyses used in the parameter evaluations were performed in accordance with the solutions of the differential equation in the first place. An exercise of this kind nevertheless serves two useful purposes: (i) to confirm that the solutions referred to, which are of closed form, are indeed reasonably reliable, and (ii) to show how closely the curves remain linear in the fatigue region, and then plateau out at the inert strength levels, $\sigma_{\mathrm{m}} P^{1 / 3}$ (fig. 2).

The equivalent construction for static fatigue is obtained from the constant stressing rate results using the rationale described earlier in the derivation of eq (13). Thus we generate the plots shown in figure 6 directly from the best-fit values of $n^{\prime}$ and $\lambda^{\prime}$ (or more strictly, via

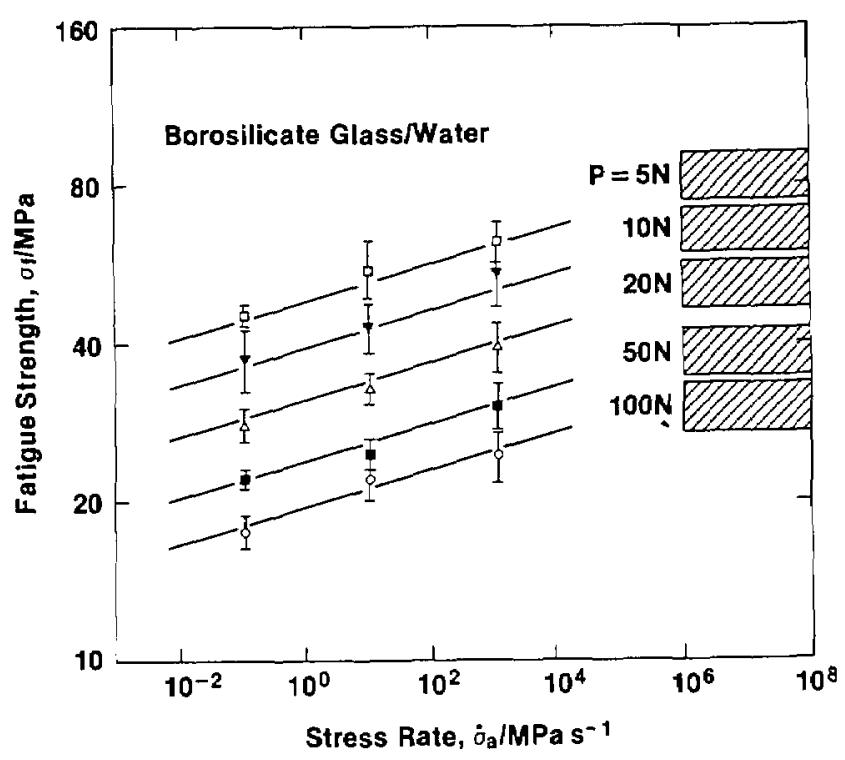

Figure 3-Dynamic fatigue responses of glasses indented at different loads. The hatched bands indicate inert strength levels. (Data courtesy of T. P. Dabbs).

eq (10), $\left.\lambda_{P}^{\prime}\right)$ determined by the data regressions shown in figures 3 and 4. Cutoff levels on the abscissa again correspond to inert strength limits. Because the construction in figure 6 is not obtained this time from regenerated solutions of the basic differential equation, we are unable to plot the curved transition between the fatigue and inert regions; however, the abruptness of the corresponding crossover points in figure 5 suggests that we may reasonably ignore any such curvature in the lifetime maps.

\section{Discussion}

\section{Quantitative Evaluation of Fracture Parameters}

The scheme presented here for reducing fatigue data to universal curves for any specified material/ 

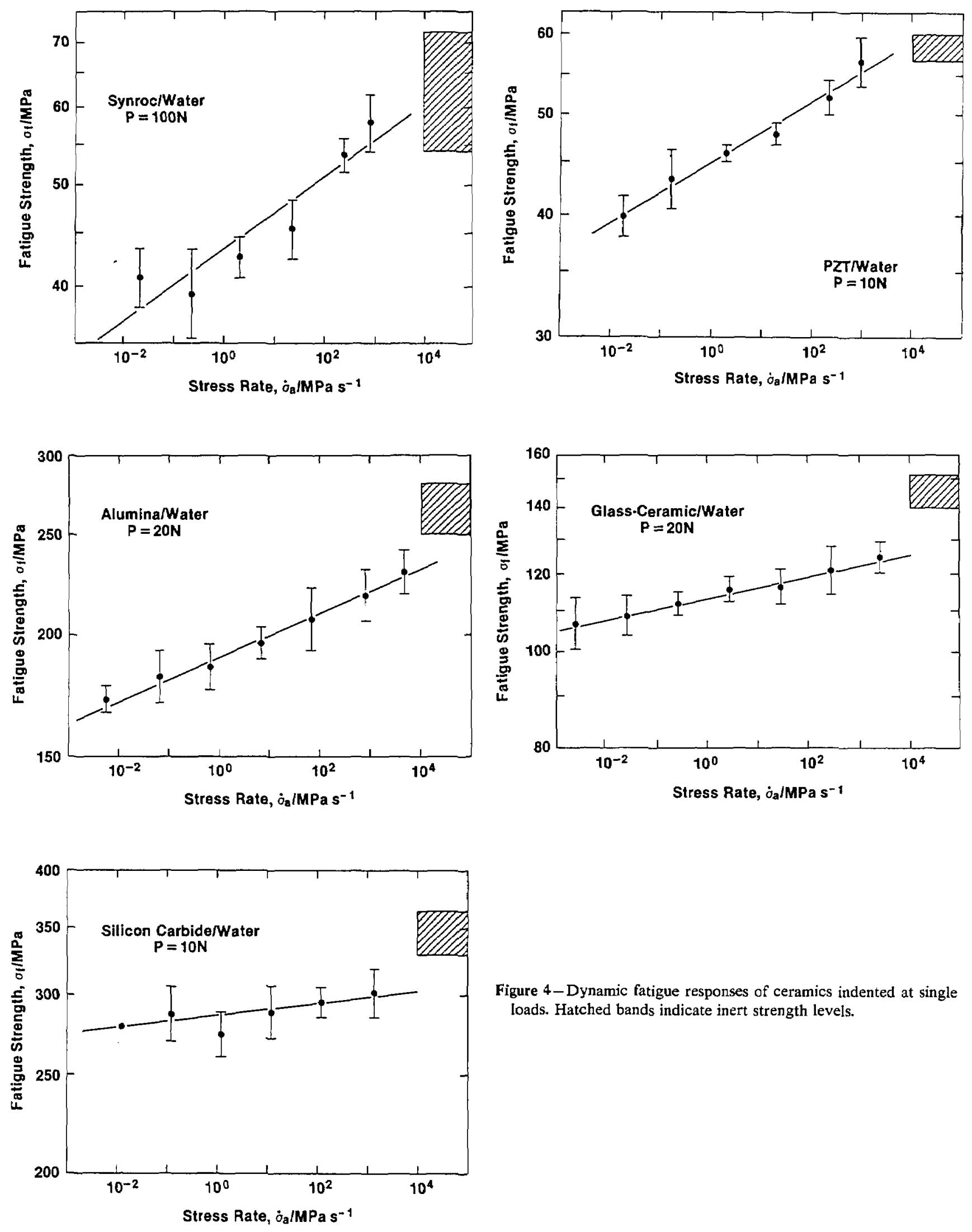

Figure 4-Dynamic fatigue responses of ceramics indented at single loads. Hatched bands indicate inert strength levels. 


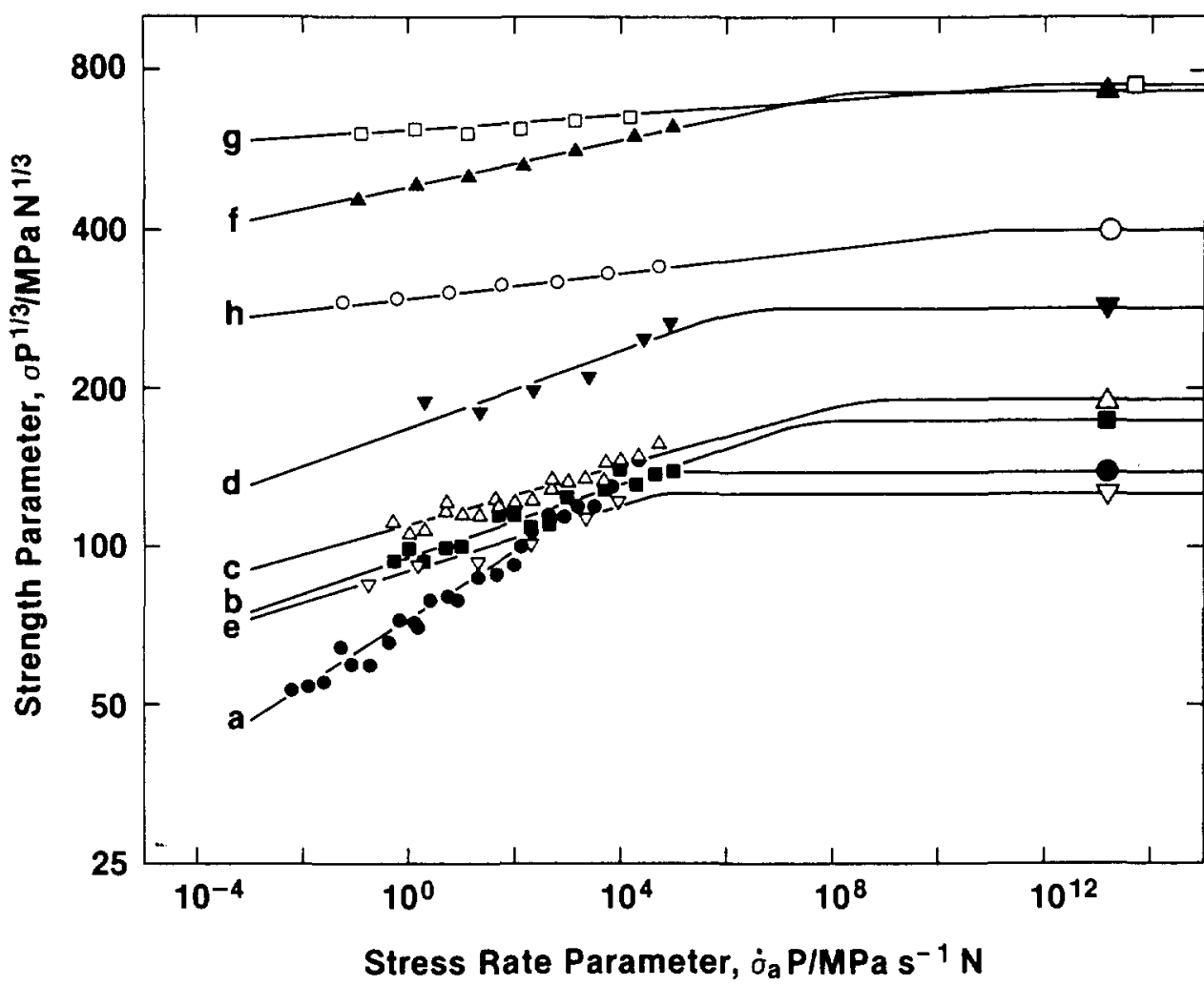

Figure 5-Dynamic fatigue master map. Error bars omitted from data points for clarity. See table 1 for key to materials.

environment system, and thence for constructing master maps to facilitate comparisons between these curves, provides an attractive route to simple, accurate, and economical evaluation of fracture parameters for design. In the following subsections we discuss how these constructions may be used as a quantitative tool for parameter determinations in different regions of the curves.

Inert Strength Levels. The position of the inert strength cutoff level, $\sigma_{\mathrm{m}} P^{1 / 3}$, may be taken as an indicator of material toughness, $K_{\mathrm{c}}$. Intrinsically tougher materials will therefore exhibit cutoffs further toward the top of a dynamic fatigue map (fig. 5) and toward the right of a static fatigue map (Fig. 6).

It should be emphasized that the correspondence implied here is not exact. To clarify this point we may invert eq (5) to obtain an explicit expression for toughness,

$$
K_{\mathrm{c}}=\left(256 \psi^{3} \xi / 27\right)^{1 / 4}(E / H)^{1 / 8}\left(\sigma_{\mathrm{m}} P^{1 / 3}\right)^{3 / 4}
$$

Thus, $K_{\mathrm{c}}$ depends on the elastic/plastic term $E / H$ as well as on $\sigma_{\mathrm{m}} P^{1 / 3}$. On the other hand, since $E / H$ varies only between 10 and 25 over the range of materials listed in table 1 , the use of an invariant, representative mean value $\left\langle(E / H)^{1 / 8}\right\rangle=1.50$ in eq (5) would lead to errors of no more than $10 \%$. Another potential source of dis- crepancy lies in the implicit assumption that geometrical similarity is preserved in the indentation pattern from material to material, as reflected in the constancy of the parameters $\xi$ and $\psi$. We have already pointed out that relaxation effects in the residual contact field can lead to reductions in the $\xi$ term. Systematically low values of $\xi$ will also be manifest in materials which deform by other than a constant-volume process or exhibit plastic pile up at the impression edges [22]. Fused silica and borosilicate glass, which tend to deform by densification [27], fall into this category, thereby explaining the tendency for the data points representing these two materials to lie above the general trend in figure 2. Finally, it has been taken as given that the radial crack patterns are always well defined, and in the materials used here this generally has been found to be so. But in materials where the microstructure is comparable in scale with the indentation event, the symmetry of the crack pattern can become severely disrupted $[8,28]$, with consequent variations in both $\xi$ and $\psi$.

It may be argued that the "effective" toughness reckoned from the cutoff position on a master map, while perhaps not an accurate measure of its macroscopically determined counterpart, nevertheless may more closely characterize the response of "natural" flaws. This is certainly likely to be so where the strength-controlling flaw in a component is created by a surface contact 


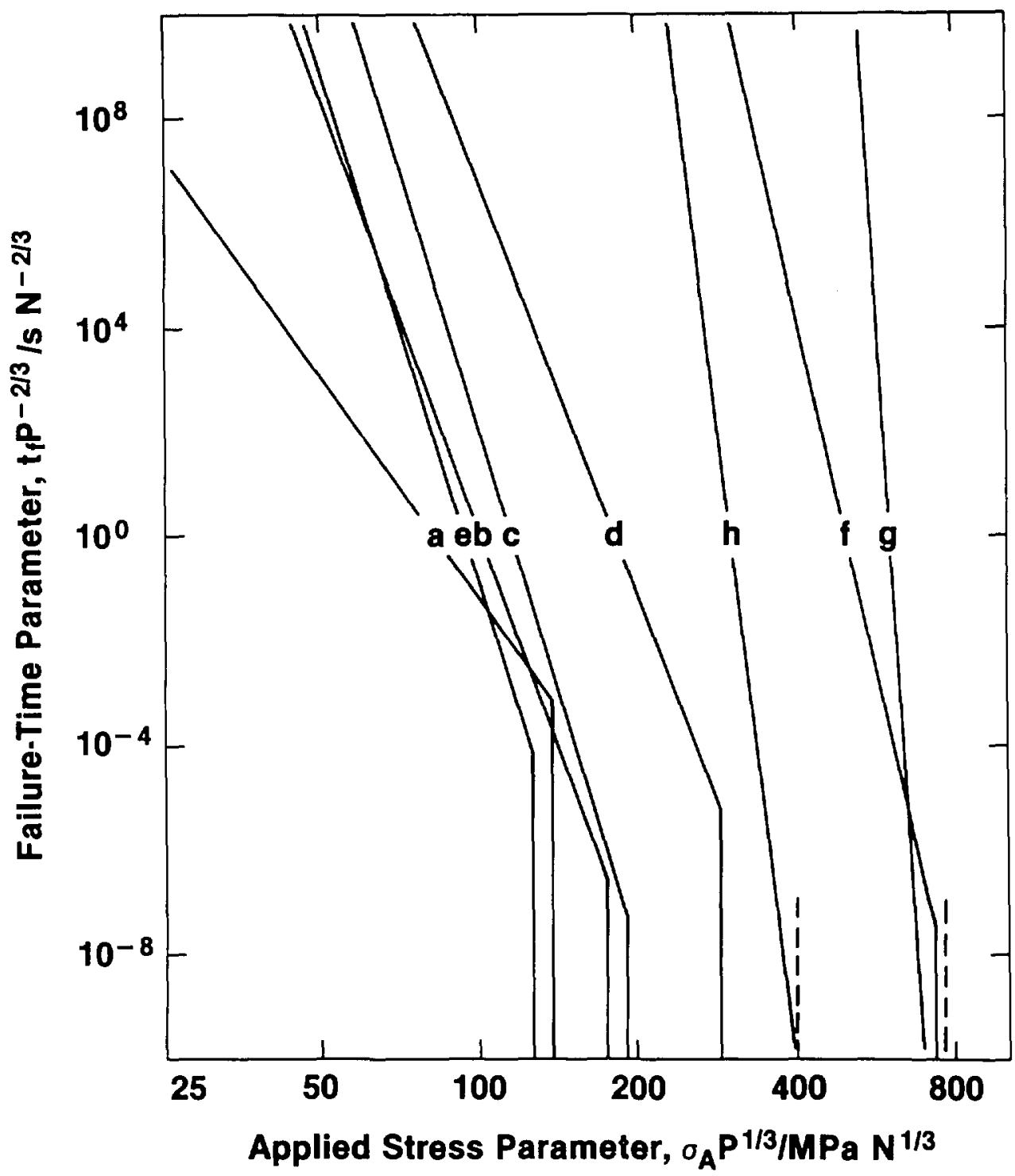

Figure 6-Static fatigue master map, generated from figure 5 . See table 1 for key to materials.

event, as in sharp-particle impact or in a machining operation. In this sense the master map approach might well be expected to serve more appropriately as a source of design parameters than the more conventional methods involving large-scale fracture specimens.

Fatigue Curve Slopes. We have noted from eqs (12) and (13) that the slope of a universal fatigue curve is a measure of the intrinsic susceptibility to slow crack growth. Thus materials with lower values of the crack velocity exponent $n$, and hence of $n^{\prime}$, eq (9a), will have greater slopes on dynamic fatigue master maps and, conversely, lower (negative) slopes on static fatigue maps.

As with the toughness, certain caution needs to be exercised when using master map data to determine $n$ values. This is because in applying the inverted form of eq (9a),

$$
n=4 n^{\prime} / 3-2 / 3,
$$

it is implicit that certain necessary conditions are met. The most important of these is the proviso $c_{0}^{\prime}<c_{\mathrm{m}}$, which we have considered at some length in this work. It is interesting to note that if this proviso is satisfied even the "anomalous" glasses which deform by non-volumeconserving processes may be analyzed in terms of eq (15); the fatigue properties are not sensitive to the origin of the residual contact field, as long as this field is of sufficient intensity to generate some precursor crack growth [11]. If such a precursor stage were not to be evident in the failure mechanics the "apparent" term $n$ ' would tend closer in value to the true $n[5,9,12,13]$. A second condition that needs to be met is that the flaws should indeed be produced in axial loading; other indentation loading systems, e.g., linear translation, give rise 
to flaws which are governed by a transformation equation with coefficients significantly different from those in eq $(15)[12,16]$.

It is seen in table 1 that the exponents obtained from this study agree well with the independent determinations for the glasses, but not for the ceramics. The relatively good agreement in the case of the glasses is attributable in part to the "model" behavior of this class of materials: transparency, isotropy, absence of microstructural complication, and ease in specimen preparation are factors which contribute to this behavior. Also, the $n$ values of the glasses are comparatively low, so fatigue effects show up more strongly. This last point, coupled with a growing realization that conventional testing techniques used to obtain macroscopic velocity data are themselves subject to uncertainty (particularly the double torsion specimen [29]), could account for the discrepancies evident in the data for the ceramics.

Fatigue Curve Intercepts. The intercept terms in the master map representations do not have such a simple interpretation in terms of basic fracture parameters. This is clear from eq (11); $\lambda_{\mathrm{p}}^{\prime}$ is a function of several quantities. Given the fatigue slope and inert strength evaluations as described in the two previous subsections, along with a direct measurement of the critical flaw size $c_{\mathrm{m}}, \lambda_{\mathrm{P}}^{\prime}$ effectively determines the crack velocity coefficient $v_{0}$. Due to the compounding of errors (particularly from the $n^{\prime}$ exponent), determinations of this kind are subject to gross uncertainty. There accordingly seems to be little value in trying to retain $v_{0}$ as a design parameter, particularly since the $\lambda^{\prime}$ terms, which can usually be determined to within $15 \%$ from dynamic fatigue data, may be used directly in lifetime formulae. In studies of the basic physics and chemistry of crack growth, of course, $v_{0}$ remains a useful coefficient for scientific analysis.

\section{Practical Implications of Master Maps}

The major appeal of the master map construction advocated here lies in the provision of a graphic indicator of the intrinsic toughness and fatigue properties of brittle materials. Each material is represented by a universal curve, the relative position of which determines the merit of that material for structural applications. The marked superiority of such materials as silicon carbide and alumina become vividly apparent in the maps of figures 5 and 6. Useful distinctions may also be made between materials which cross over within the data range, e.g., soda-lime glass and PZT. On the basis of straight inert-strength testing we might reckon the first of these as the stronger material, whereas for applica- tions involving sustained stresses it is the second which would tend to the larger lifetimes. Such crossovers would not be so obvious from the raw fracture mechanics parameters. It will be appreciated that this kind of intercomparison is made on the basis of "equivalent" flaw sizes: in this respect the indentation method, through its control over the flaw severity via the contact load, is unique in its capacity for reducing strength data to a common denominator.

In arguing the merits of this approach we do not mean to imply that it is only the intrinsic fracture properties which play an important role in the determination of component strengths and lifetimes; the effective sizes of the naturally occurring flaws which ultimately cause failure must also be known. Our procedure, by introducing flaws greater in severity than any of these natural flaws, automatically excludes information concerning the latter from the data. What our scheme effectively allows us to do is to determine the intrinsic parameters in a truly independent manner. All necessary extrinsic flaw parameters should be obtainable from straightforward inert strength tests (run at a single stressing rate), in the form of the usual statistical distribution functions. Lifetime predictions for as-prepared components could then be made without ever having to accumulate vast quantities of fatigue data [2]. In adopting this strategy one needs to keep in mind the strong influence that any persisting residual stress concentrations associated with the original initiation processes (in our case the elastic/plastic deformation) might exert on the subsequent flaw evolution. In the absence of information as to this aspect of flaw characterization steps should be taken to design conservatively, on the basis of "worst-case" configurations wherever possible. This last point is dealt with in greater detail in reference [25].

It has been indicated at several points that the existence of any spurious stresses incurred during the mechanical, chemical, or thermal history of a material would necessitate a third contribution to the starting stress intensity factor in eq (1), with consequent deviations from the currently determined toughness and fatigue relations. The fact that such deviations were not observed in the materials studied here may be taken as evidence that this potential complication has been successfully avoided. Again, it may be well to emphasize that it may not be so simple to confirm the elimination of spurious stresses from surfaces whose strengths are controlled by natural flaws, particularly in materials with typically wide flaw distributions; nor, of course, may we wish to eliminate them, bearing in mind that these stresses are most often compressive.

Finally, a comment may be made concerning the convenience of indentation load as a variable for in- 
vestigating fundamental flaw size effects. By systematically reducing the load we can produce corresponding smaller flaws, thereby providing a link between macroscopic and microscopic crack behavior. Any change in the nature of the indentation flaw will then become evident as deviations from universal plots, much as just described in relation to the spurious stress influence. In this way it has been possible to demonstrate that indentation flaws in glasses undergo an abrupt transition in properties below a threshold load (corresponding to a flaw size $\approx \mu \mathrm{m}$ ): above this threshold the macroscopically determined laws of crack growth remain perfectly valid, recardless of scale, provided that the residual contact term is duly accounted for [11]; below the threshold the universal curves no longer apply, and failure becomes dominated by initiation micromechanics $[17,30]$. The indentation technique should prove similarly useful for studying size effects in ceramics, particularly for polycrystalline materials with relatively coarse microstructures.

\section{Conclusions}

1) The indentation-flaw technique provides an attractive route to the evaluation of intrinsic fracture parameters. Coupled with independent determinations of natural flaw distributions, the approach offers the prospect of accurate lifetime predictions with optimum specimen economy.

2) The control over the nature, shape, and above all, the size (via the contact load) of the indentation flaw allows for the derivation of a universal fracture formulation. Each material is represented by a single curve which incorporates the toughness and fatigue properties. Composite plots of these curves produce master maps, affording a simple graphic format for materials comparisons.

3) The inert strength cutoff on such a master map is a measure of effective material toughness. For "wellBehaved" materials this effective toughness is consistent with macroscopically measured $K_{\mathrm{c}}$ values. In cases where inconsistency is observed the toughness reckoned from indentation data may provide a more reliable indication of the response of the typical natural flaw.

4) The slope of the fatigue curve on a master map is a measure of the susceptibility of a material to subcritical crack growth. The crack velocity exponent determined from this slope is an apparent value, $n^{\prime}$, which is converted to the true value, $n$, via a simple transformation equation.
5) Deviations from universality on a master map indicate an extraneous influence in the fracture mechanics, e.g., spurious stress states, microstructure/crack interactions, and threshold size effects.

The authors thank T. P. Dabbs for providing raw fracture data on the glasses, and L. Respall and S. J. Mann for their help with specimen preparation.

\section{References}

[1] Wiederhorn, S. M.; E. R. Fuller, J. Mandel, and A. G. Evans. J. Amer. Ceram. Soc., Vol. 59, 1976, pp. 404-411.

[2] Ritter, J. E.; N. Bandyopadhyay and K. Jakus., Ceram. Bull., Vol. 60, 1981, pp. 798-806.

[3] Marshall, D. B., and B. R. Lawn, J. Mater. Sci., Vol. 14, 1979, pp. 2001-2012.

[4] Marshall, D. B.; B. R. Lawn and P. Chantikul, ibid., Vol. 14, 1979, pp. 2225-2235.

[5] Marshall, D. B. and B. R. Lawn, J. Amer. Ceram. Soc., Vol. 63, 1980, pp. 532-536.

[6] Chantikul, P,; B. R. Lawn and D. B. Marshall, ibid., Vol. 64, 1981, 322-325.

[7] Anstis, G. R.; P. Chantikul, B. R. Lawn, and D. B. Marshall, ibid., Vol. 64, pp. 533-538.

[8] Chantikul, P.; G. R. Anstis, B. R. Lawn, and D. B. Marshall, ibid., Vol. 64, pp. 539-543.

[9] Lawn, B. R.; D. B. Marshall, G. R. Anstis, and T. P. Dabbs, $J$. Mater. Sci., Vol. 16, 1981, pp. 2846-2854

[10] Cook, R. F.; B. R. Lawn and G. R. Anstis., ibid., Vol. 17, 1982, pp. 1108-1116.

[11] Dabbs, T. P.; B. R. Lawn and P. L. Kelly, Phys. Chem. Glasses, Vol. 23, 1982, pp. 58-66.

[12] Symonds, B. L.; R. F. Cook and B. R. Lawn, J. Mater. Sci, in press.

[13] Marshall, D. B. and B. R. Lawn. J. Amer. Ceram. Soc., Vol. 64, 1981, pp. C6-C7

[14] Marshall, D. B.; A. G. Evans, B. T. Khuri-Yakub, J. W. Tien, and G. S. King, to be published.

[15] Lawn, B. R., in Fracture Mechanics of Ceramics, R. C. Bradt, A. G. Evans, D. P. H. Hasselman and F. F. Lange, Eds., Plenum, New York, 1983, Vol. 5, pp. 1-25.

[16] Fuller, E. R.; B. R. Lawn and R. F. Cook, J. Amer. Ceram. Soc. Vol. 66, 1983, pp. 314-321.

[17] Dabbs, T. P., and B. R. Lawn, Phys. Chem. Glasses, Vol. 23, 1982, pp. 93-97.

[18] Cook, R. F., unpublished work.

[19] Marshall, D. B. and B. R. Lawn, J. Amer. Ceram. Soc, Vol. 61, 1978, pp. 21-27.

[20] Mould, R. E., and R. D. Southwick, ibid., Vol. 42, 1959, pp. $542-547,582-592$.

[21] Lawn, B. R. and E. R. Fuller, J. Mater. Sci, , Vol. 10, 1975, pp. 2016-2024.

[22] Lawn, B. R.; A. G. Evans and D. B. Marshall, J. Amer. Ceram. Soc., Vol. 63, 1980, pp. 574-581. 
[23] Wiederhorn, S. M., in Fracture Mechanics of Ceramics, R. C. Bradt, D. P. H. Hasselman and F. F. Lange, Eds., Plenum, New York, 1974, vol. 2, pp. 613-646.

[24] Flexure Testing of Glass, A.S.T.M. Annual Book of Standards, ASTM, Philadelphia, 1979, Part 17, C158-C172.

[25] Gonzalez, A. C.; H. Multhopp, R. F. Cook, B. R. Lawn, and S. W. Frieman, a paper in Methods for Assessing the Structural Reliability of Brittle Materials, ASTM Special Technical Publication, Philadelphia (in press).

[26] Lawn, B. R.; D. B. Marshall, P. Chantikul, and G. R. Anstis, J. Austral. Ceram. Soc., Vol. 16, 1980, pp. 4-9.

[27] Arora, A.; D. B. Marshall, B. R. Lawn, and M. V. Swain, $J$. Non-Cryst. Solids, Vol. 31, 1979, pp. 415-428.

[28] Smith, S. S., and B. J. Pletka, in Fracture Mechanics of Ceramics, R. C. Bradt, A. G. Evans, D. P. H. Hasselman and F. F. Lange, Eds., Plenum, New York, 1983, Vol. 6, pp. 189-210.

[29] Pletka, B. J.; E. R. Fuller and B. G. Koepke, in Fracture Mechanics Applied to Brittle Materials, S. W. Freiman, Ed., A.S.T.M. Special Technical Publication 678, ASTM., Philadelphia, 1978, pp. 19-37.

[30] Dabbs, T. P.; C. J. Fairbanks and B. R. Lawn, a paper in Methods for Assessing the Structural Reliability of Brittle Materials, ASTM Special Technical Publication. Philadelphia (in press).

[31] Wiederhorn, S. M, J. Amer. Ceram. Soc., Vol. 52, 1969, pp. 99-105.

[32] Wiederhorn, S. M., unpublished work.

[33] Ritter, J. E., and C. L. Sherbourne, J. Amer. Ceram. Soc., Vol. 54, 1971, pp. 601-605.

[34] Cook, R. F.; B. R. Lawn, T. P. Dabbs, K. D. Reeve, E. J. Ramm, and J. L. Woolfrey, J. Amer, Ceram. Soc., Vol, 65, 1982, pp. C172-C173.

[35] Gonzalez, A. C., and S. W. Freiman, unpublished work.

[36] McHenry, K. D.; T. Yonushonis and R. E. Tressler. J. Amer. Ceram. Soc, Vol. 59, 1976, pp. 262-263.

[37] Koepke, B. G. Unpublished work.

[38] Pletka, B. J., and S. M. Wiederhorn. J. Mater. Sc., Vol. 17, 1982, pp. 1247-1268. 